\title{
Alterações clínicas e hematológicas em tamanduá-bandeira (Myrmecophaga tridactyla) vítima de incêndios no Pantanal Mato-grossense: Relato de caso
}

Clinical and hematological changes in giant anteaters (Myrmecophaga tridactyla) victims of fires in the Pantanal Mato-grossense: Case report

Cambios clínicos y hematológicos en osos hormigueros gigantes (Myrmecophaga tridactyla) víctimas de incendios en el Pantanal Mato-grossense: Reporte de caso

Thays Guimarães de Souza ORCID: https://orcid.org/0000-0001-7920-7474 Universidade Federal do Mato Grosso, Brasil E-mail: thays.veterinaria@outlook.com.br Marisol Alves de Barros ORCID: https://orcid.org/0000-0002-6720-0053 Universidade Federal do Mato Grosso, Brasil E-mail: soolbarros@gmail.com Carolina Zorzo

ORCID: https://orcid.org/0000-0001-7635-1374 Universidade Federal do Mato Grosso, Brasil E-mail: carolzorzo6@gmail.com

Jaqueline Camargo Borges ORCID: https://orcid.org/0000-0003-3533-7618 Universidade Federal do Mato Grosso, Brasil E-mail: jaquelineborges13@hotmail.com

Tayane Bruna Soares Magalhães ORCID: https://orcid.org/0000-0002-4199-6663 Universidade Federal do Mato Grosso, Brasil E-mail: tayane.bruna16@hotmail.com

Adriane Jorge Mendonça ORCID: https://orcid.org/0000-0002-9367-5028 Universidade Federal do Mato Grosso, Brasil E-mail: adrianejorge.m@gmail.com

João Gabriel Matheiski Alkmim ORCID: https://orcid.org/0000-0001-5962-4924 Universidade Federal do Mato Grosso, Brasil E-mail: gabrielmalkmim@hotmail.com

Thaís Oliveira Morgado

ORCID: https://orcid.org/0000-0002-2974-3241 Universidade Federal do Mato Grosso, Brasil E-mail: thaismorgado@gmail.com

Antonio Henrique Kukzmarski ORCID: https://orcid.org/0000-0003-1347-3467 Universidade Federal do Mato Grosso, Brasil E-mail: ah.kucz@gmail.com

Ana Letícia Prata Palermo

ORCID: https://orcid.org/0000-0002-6926-6881

Universidade Federal do Mato Grosso, Brasil

E-mail: analeticiapalermo@outlook.com

\section{Resumo}

As queimadas que ocorreram no ano de 2020 no Pantanal trouxeram intensos prejuízos à fauna e flora da região, sendo que o tamanduá-bandeira foi um dos mamíferos mais afetados pelas queimadas. O presente trabalho teve como objetivo descrever alterações hematológicas encontradas nos exames laboratoriais de um tamanduá-bandeira (Myrmecophaga tridactyla), vítima dos incêndios ocorridos no Pantanal mato-grossense, correlacionando-as ao quadro clínico do animal. O paciente foi resgatado no mês de setembro de 2020 com lesões graves por queimaduras nas regiões de membros e face. Após quatro dias de internação (D4) foi feita colheita de sangue para a realização de hemograma e bioquímica sérica. Notou-se diminuição nos valores de eritrócitos $\left(0,98 \times 10^{6} / \mathrm{mm}^{3} ; 2,05-2,67\right.$ $\left.\mathrm{x} 10^{6} / \mathrm{mm}^{3}\right)$, hemoglobina $(4,8 \mathrm{~g} / \mathrm{dl} ; 10,6-12,9 \mathrm{~g} / \mathrm{dl})$ e hematócrito $(16 \% ; 35,44-40,1 \%)$, contagem total de leucócitos dentro dos valores de normalidade $\left(16,0 \times 10^{3} / \mathrm{mm}^{3} ; 5,59-18,14 \times 10^{3} / \mathrm{mm}^{3}\right)$, com desvio à esquerda 
degenerativo, presença de metamielócitos $\left(0,7 \times 10^{3} / \mathrm{mm}^{3} ; 0-0 \times 10^{3} / \mathrm{mm}^{3}\right)$, bastonetes $\left(5,2 \times 10^{3} / \mathrm{mm}^{3} ; 0-0,06 \times\right.$ $\left.10^{3} / \mathrm{mm}^{3}\right)$ e segmentados $\left(5,1 \times 10^{3} / \mathrm{mm}^{3} ; 3,91-3,88 \times 10^{3} / \mathrm{mm}^{3}\right)$. Os eosinófilos $\left(3,4 \times 10^{3} / \mathrm{mm}^{3} ; 0,08-1,86 \mathrm{x}\right.$ $\left.10^{3} / \mathrm{mm}^{3}\right)$, monócitos $\left(0,5 \times 10^{3} / \mathrm{mm}^{3} ; 0,01-0,2 \times 10^{3} / \mathrm{mm}^{3}\right)$ e plaquetas $\left(168 \times 10^{3} / \mathrm{mm}^{3} ; 52,2-134,4 \times 10^{3} / \mathrm{mm}^{3}\right)$ estavam aumentados e os valores de proteínas plasmáticas totais diminuídos $(4,2 \mathrm{~g} / \mathrm{dl} ; 7,77-8,42 \mathrm{~g} / \mathrm{dl})$. Os valores de ALT (121 UI/L; 7,51 - 23,47 UI/L), AST (143 UI/L; 13,62 - 28,62 UI/L) e CK (13252,0 UI/L; 41,45 - 81,77 UI/L), mostraram-se significativamente aumentados e de proteínas totais diminuído (4,8 g/dl; 5,49-6,61 g/dl). Queimaduras de segundo e terceiro graus em tamanduá-bandeira podem causar desvio à esquerda degenerativo, anemia intensa, hipoproteinemia e alterações marcantes de enzimas que avaliam dano muscular. $\mathrm{O}$ impacto das queimadas no Pantanal mato-grossense no ano de 2020 precisa ser avaliado, sendo necessária a implantação de políticas públicas de combate aos incêndios mais eficazes.

Palavras-chave: Queimadura; Desvio à esquerda; Pantanal; Xenarthra.

\begin{abstract}
The fires that happened in 2020 in the Pantanal caused intense damage to the fauna and flora of the region, and the giant anteater was one of the most affected mammals by the fires. The present study aimed to describe hematological changes found in laboratory tests of a giant anteater (Myrmecophaga tridactyla), victim of the fires that occurred in the Pantanal of Mato Grosso, correlating with the animal's clinical condition. The patient was rescued in September 2020 with serious injuries from burns in the limbs and face. After four days of hospitalization (D4), blood was collected for blood count and serum biochemistry.. A decrease in erythrocyte values was noted $\left(0.98 \times 10^{6} / \mathrm{mm}^{3} ; 2.05\right.$ $\left.-2.67 \times 10^{6} / \mathrm{mm}^{3}\right)$, hemoglobin $(4.8 \mathrm{~g} / \mathrm{dl} ; 10.6-12.9 \mathrm{~g} / \mathrm{dl})$ and hematocrit $(16 \% ; 35.44-40.1 \%)$, total white blood cell count within the normal range $\left(16.0 \times 10^{3} / \mathrm{mm}^{3} ; 5.59-18.14 \times 10^{3} / \mathrm{mm}^{3}\right)$, degenerative left shift, with presence of metamielocytes $\left(0.7 \times 10^{3} / \mathrm{mm}^{3} ; 0-0 \times 10^{3} / \mathrm{mm}^{3}\right)$, rods $\left(5.2 \times 10^{3} / \mathrm{mm}^{3} ; 0-0.06 \times 10^{3} / \mathrm{mm}^{3}\right)$ and segmented $(5.1 \times$ $\left.10^{3} / \mathrm{mm}^{3} ; 3.91-3.88 \times 10^{3} / \mathrm{mm}^{3}\right)$. Eosinophils $\left(3.4 \times 10^{3} / \mathrm{mm}^{3} ; 0.08-1.86 \times 10^{3} / \mathrm{mm}^{3}\right)$, monocytes $\left(0.5 \times 10^{3} / \mathrm{mm}^{3}\right.$; $\left.0.01-0.2 \times 10^{3} / \mathrm{mm}^{3}\right)$ and platelets $\left(168 \times 10^{3} / \mathrm{mm}^{3} ; 52.2-134.4 \times 10^{3} / \mathrm{mm}^{3}\right)$ were increased and the total plasma protein values decreased $(4.2 \mathrm{~g} / \mathrm{dl} ; 7.77-8.42 \mathrm{~g} / \mathrm{dl})$. ALT (121 UI/L; $7.51-23.47 \mathrm{UI} / \mathrm{L})$, AST (143 UI/L; $13.62-$ 28.62 UI/L) and CK (13252.0 UI/L; 41.45 - 81.77 UI/L) were significantly increased and total protein decreased (4.8 $\mathrm{g} / \mathrm{dl} ; 5.49-6.61 \mathrm{~g} / \mathrm{dl})$. Second and third degree burns in giant anteaters cause degenerative deviation, severe anemia, hypoproteinemia and marked changes in enzymes that assess muscle damage. The impact of fires in the Pantanal of Mato Grosso in 2020 needs to be assessed, requiring the implementation of more effective public policies to combat fires.
\end{abstract}

Keywords: Burn; Left shift; Pantanal; Xenarthra.

\title{
Resumen
}

Los incendios ocurridos en el año 2020 en el Pantanal provocaron daños intensos a la fauna y flora de la región, y el oso hormiguero gigante fue uno de los mamíferos más afectados por los incendios. El presente estudio tuvo como objetivo describir los cambios hematológicos encontrados en las pruebas de laboratorio de un oso hormiguero gigante (Myrmecophaga tridactyla), víctima de los incendios ocurridos en el Pantanal de Mato Grosso, correlacionados con la condición clínica del animal. El paciente fue rescatado en septiembre de 2020 con heridas graves por quemaduras en las extremidades y la cara. Después de cuatro días de hospitalización (D4), se recolectó sangre para hemograma y bioquímica sérica. Se notó una disminución en los valores de eritrocitos $\left(0,98 \times 10^{6} / \mathrm{mm}^{3} ; 2,05-2,67\right.$ x $\left.10^{6} / \mathrm{mm}^{3}\right)$, hemoglobina $(4,8 \mathrm{~g} / \mathrm{dl} ; 10,6-12,9 \mathrm{~g} / \mathrm{dl})$ y hematocrito $(16 \% ; 35,44-40,1 \%)$, recuento total de glóbulos blancos dentro del rango normal $\left(16,0 \times 10^{3} / \mathrm{mm}^{3} ; 5,59-18,14 \times 10^{3} / \mathrm{mm}^{3}\right)$, desplazamiento degenerativo a la izquierda, con presencia de metamielocitos $\left(0,7 \times 10^{3} / \mathrm{mm}^{3} ; 0-0 \times 10^{3} / \mathrm{mm}^{3}\right)$, varillas $\left(5,2 \times 10^{3} / \mathrm{mm}^{3} ; 0-0,06 \times 10^{3} / \mathrm{mm}^{3}\right)$ y segmentadas $\left(5,1 \times 10^{3} / \mathrm{mm}^{3} ; 3,91-3,88 \times 10^{3} / \mathrm{mm}^{3}\right)$. Los eosinófilos $\left(3,4 \times 10^{3} / \mathrm{mm}^{3} ; 0,08-1,86 \times 10^{3} / \mathrm{mm}^{3}\right), \mathrm{los}^{3}$ monocitos $\left(0,5 \times 10^{3} / \mathrm{mm}^{3} ; 0,01-0,2 \times 10^{3} / \mathrm{mm}^{3}\right)$ y las plaquetas $\left(168 \times 10^{3} / \mathrm{mm}^{3} ; 52,2-134,4 \times 10^{3} / \mathrm{mm}^{3}\right)$ aumentaron y los valores de proteínas plasmáticas totales disminuyeron $(4,2 \mathrm{~g} / \mathrm{dl} ; 7,77-8,42 \mathrm{~g} / \mathrm{dl})$. Los valores de ALT (121 UI/L; 7,51 - 23,47 UI/L), AST (143 UI/L; 13,62 - 28,62 UI/L) y CK (13252,0 UI/L; 41,45 - 81,77 UI/L) aumentaron significativamente y la proteína total disminuyó $(4,8 \mathrm{~g} / \mathrm{dl} ; 5,49-6,61 \mathrm{~g} / \mathrm{dl})$. Las quemaduras de segundo y tercer grado en osos hormigueros gigantes causan desviación degenerativa, anemia severa, hipoproteinemia y cambios marcados en las enzimas que evalúan el daño muscular. Es necesario evaluar el impacto de los incendios en el Pantanal de Mato Grosso en el año 2020, lo que requiere la implementación de políticas públicas más efectivas para combatir los incendios.

Palabras clave: Quemar; Cambio a la izquierda; Pantanal; Xenarthra.

\section{Introdução}

O tamanduá-bandeira (Myrmercophaga tridactyla) pertence a superordem Xenarthra, ordem Pilosa, do qual também fazem parte as preguiças, o tamanduá mirim (Tamandua tetradactyla) e o tamanduaí (Cyclopes didactylus) (Miranda, 2014). A 
sua distribuição geográfica é ampla, sendo encontrado no Panamá, Nicarágua, Honduras, El Salvador, Colômbia, Venezuela, Equador (leste do Andes), Guianas, Paraguai, Peru, Bolívia, Argentina, e em todos os biomas brasileiros (Miranda et al., 2014).

Por ter pelos longos mais propícios a queimar, e pela locoção mais lenta, o tamanduá-bandeira é um dos mamíferos mais afetados pelas queimadas, sua pouca mobilidade é atribuída principalmente ao seu grande porte e hábitos alimentares com ingestão de baixa quantidade de calorias, baixo índice metabólico e baixa temperatura corporal (Ribeiro et al., 2016). Além disso é conhecido que M. tridactyla possui baixa acurácia visual e auditiva (Miranda, 2014).

Alguns aspectos reprodutivos como a maturidade sexual próxima a três anos, a reprodução apenas uma vez ao ano devido a um longo período gestacional, além de outros fatores como a destruição de ambientes naturais e traumas por atropelamento, levaram a um rápido declínio populacional nos últimos anos. Atualmente essa espécie é considerada vulnerável de acordo com o ICMbio (Instituto Chico Mendes de Conservação da Biodiversidade) (Miranda et al., 2015) e está na lista vermelha de espécies ameaçadas de extinção da International Union for Conservation of Nature (IUCN) (Miranda et al., 2014).

O ano de 2020 foi marcado com a pior seca registrada no Pantanal em 60 anos. Na estação chuvosa houve queda de 57\% na pluviosidade, elevando os limiares de inflamabilidade ao seu mais alto nível desde 1980, ocasionando a perda de quase um terço do Pantanal decorrente das queimadas (Libonati et al., 2020). Dados do Instituto Nacional de Pesquisas Espaciais (INPE) referente ao período de 1998 a 2020 mostram que este último ano registrou 22.116 focos de incêndio no bioma, o valor mais alto em 22 anos e com um aumento de 210\% se comparado ao ano de 2019. Relatórios do INPE também apontaram que os meses com maiores registros de focos de queimadas de 2020 foram setembro e outubro.

O presente trabalho teve como objetivo descrever alterações hematológicas encontradas nos exames laboratoriais de um tamanduá-bandeira (M. tridactyla), vítima dos incêndios ocorridos no Pantanal mato-grossense, correlaciona-las ao quadro clínico do animal.

\section{Metodologia}

Foi encaminhado ao Hospital Veterinário da Universidade Federal do Mato Grosso (HOVET - campus Cuiabá) um tamanduá-bandeira (Myrmercophaga tridactyla) adulto, fêmea, pesando 21,4 kg, resgatado no Pantanal Mato-grossense no mês de setembro, com queimaduras nos quatro membros (Figura 1) além de laceração nos membros torácicos com exposição óssea e presença de mí́ase. Ao exame físico notou-se subnutrição, prostração e desidratação (10\%). Fluidoterapia intravenosa emergencial foi instituída imediatamente após a admissão hospitalar, utilizando-se solução de Ringer lactato na dose de $15 \mathrm{ml} / \mathrm{kg}$. 
Figura 1. Tamanduá-bandeira (Myrmercophaga tridactyla), resgatado do Pantanal Mato-grossense com queimaduras nos membros e na face atendido no Hospital Veterinário da Universidade Federal do Mato Grosso - campus Cuiabá.

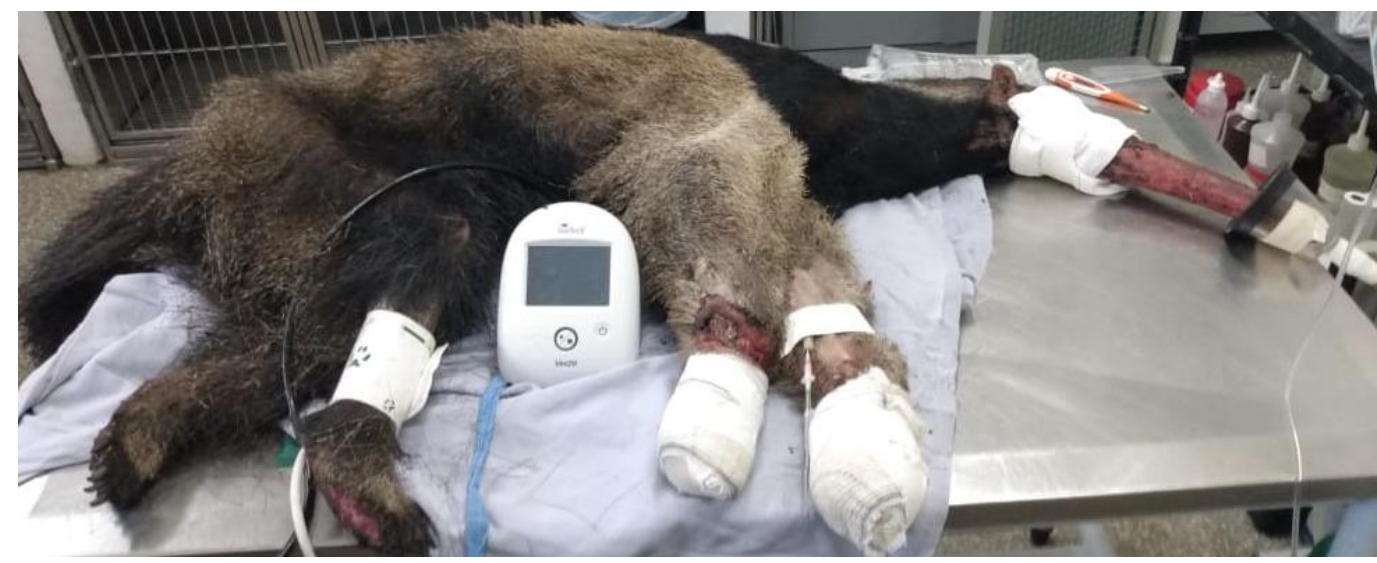

Fonte: Hospital Veterinário da Universidade Federal do Mato Grosso - campus Cuiabá (2020).

A porcentagem de superfície corporal queimada foi estimada utilizando-se a "regra de 9", que consiste numa adaptação da técnica utilizada para pacientes humanos. Para áreas específicas da superfície corporal são atribuídas as seguintes porcentagens: $9 \%$ para cada membro torácico, $18 \%$ para cada membro pélvico, $9 \%$ para a cabeça, $1 \%$ para o pescoço, $18 \%$ para o dorso e $18 \%$ para o tronco do corpo ventral (Merk \& Miller, 2013). Dessa forma estimou-se que cerca de $63 \%$ do corpo do paciente apresentava queimaduras.

O paciente foi submetido a procedimento anestésico para coleta de sangue, limpeza, escarificação e curativo das feridas. A medicação pré-anestésica foi realizada com Midazolan (Midazolan® 0,3 mg/kg, IM. Cloridrato de Midazolam, Hipolabor, Sabará, MG, Brasil), Cloridrato de Metadona (MYTedom® 0,2 mg/kg, IM. Cristália, Itapira, SP, Brasil) e Cloridrato de Cetamina 10\% (Cetamin® $8 \mathrm{mg} / \mathrm{kg}$, IM. Syntec, Santana de Parnaíba, SP, Brasil). Associado a anestesia intravenosa, foi feito bloqueio anestésico do plexo braquial com Lidocaína (Lidovet® $9 \mathrm{mg} / \mathrm{kg}$. Bravet, Brasil).

Durante o procedimento, o paciente apresentou hipotensão (Pressão Arterial Média [PAM] $50 \mathrm{mmHg} ; 80$ - 116 $\mathrm{mmHg}$ ), considerando os valores de referência encontrado para preguiças (Bradypus variegatus), membros da ordem Pilosa, da qual também fazem parte os tamanduás (Duarte et al., 2003). Portanto, foi necessária a administração de Sulfato de Efedrina (Efedrin®. Cristália, Itapira, SP, Brasil) em dois bolus $(0,05 \mathrm{mg} / \mathrm{kg}$, IV e $0,1 \mathrm{mg} / \mathrm{kg}$, IV) e infusão contínua de Cloridrato Dopamina (Cloridrato de Dopamina ${ }^{\circledR} 5 \mathrm{mcg} / \mathrm{kg} / \mathrm{min}$, IV. Hipolabor, Sabará, MG, Brasil) para tentar reverter o quadro de hipotensão. Ao final do procedimento o paciente permaneceu hipotenso, sendo necessária instituição de infusão contínua intravenosa de Hemitartarato de Norepinefrina (Hyponor® 0,1 mcg/kg/min; 0,2 mcg/kg/min; 0,36 mcg/kg/min. Hypofarma, Ribeirão das Neves, MG, Brasil).

Durante os 12 dias de internação, foram realizados exames laboratoriais em dois momentos: o primeiro no dia em que o paciente deu entrada no HOVET (D0) e o outro, quatro dias após (D4). A colheita foi realizada por venopunção da veia cefálica e o sangue foi acondicionado em tubo com Ácido Etilenodiamino Tetra-Acético (Ethylenediamine Tetraacetic Acid EDTA) (2mL), para a realização do hemograma e em tubo com ativador de coágulo para realização da bioquímica sérica. As amostras foram processadas de acordo com as técnicas descritas por Weiser (2015). Os resultados dos hemogramas e bioquímicas séricas estão distribuídos nas Tabelas 1 e 2 respectivamente.

No hemograma realizado no D0 observou-se anemia, hipoproteinemia (5,2 g/dl) e, apesar de não haver anormalidade na leucometria global $\left(14,1 \times 10^{3} / \mathrm{mm}^{3}\right)$, foi constatado intenso desvio à esquerda degenerativo, com presença de metamielócitos $\left(8,9 \times 10^{3} / \mathrm{mm}^{3}\right)$, bastonetes $\left(2,7 \times 10^{3} / \mathrm{mm}^{3}\right)$ e raros segmentados $\left(0,1 \times 10^{3} / \mathrm{mm}^{3}\right)$. 
No D4 houve agravo da anemia comparado ao exame anterior (Tabela 1). Com relação a contagem total de leucócitos, notou-se discreto aumento, porém o valor se manteve dentro da normalidade. $\mathrm{O}$ animal permaneceu com desvio à esquerda degenerativo, apresentando eosinofilia, monocitose e trombocitose. Os valores de proteínas plasmáticas totais continuavam diminuídos. Os outros resultados estavam de acordo com os parâmetros estabelecidos para a espécie.

Tabela 1. Comparativo dos hemogramas de tamanduá-bandeira (Myrmecophaga tridactyla) atendido no Hospital Veterinário da Universidade Federal de Mato Grosso, com lesões de queimaduras térmicas realizados no dia da internação (D0) e após 4 dias de internação (D4).

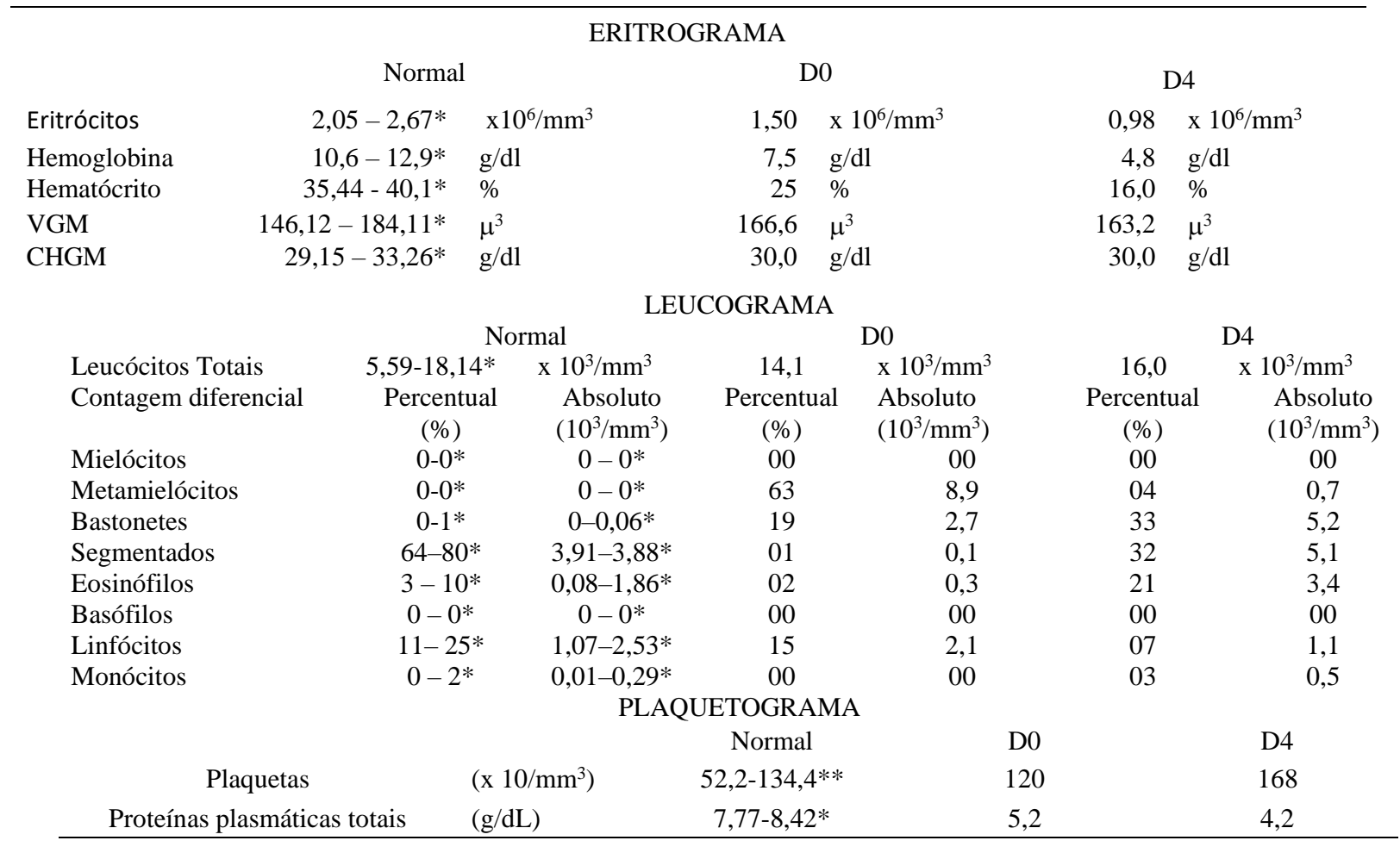

* Valores de referência para a espécie segundo F. Miranda (2014). ** Valores de referência para a espécie segundo Nucci, et al. (2014). Fonte: Autores.

Tabela 2. Comparativo da bioquímica sérica de tamanduá-bandeira (Myrmecophaga tridactyla) atendido no Hospital Veterinário da Universidade Federal de Mato Grosso, com lesões de queimaduras térmicas realizados no dia da internação (D0) e após 4 dias de internação (D4).

\begin{tabular}{lccccc}
\hline \multicolumn{1}{c}{ Exame } & Normal & & D0 & D4 & \\
\hline Ureia & $35,18-71,74^{*}$ & $\mathrm{mg} / \mathrm{dl}$ & 92,0 & 61,0 & $\mathrm{mg} / \mathrm{dl}$ \\
Creatinina & $0,68-1,42^{*}$ & $\mathrm{mg} / \mathrm{dL}$ & 1,2 & 0,8 & $\mathrm{mg} / \mathrm{dL}$ \\
Alanina Amino Transferase (ALT) & $7,51-23,47^{*}$ & $\mathrm{UI} / \mathrm{L}$ & 62,0 & 121,0 & $\mathrm{UI} / \mathrm{L}$ \\
Aspartato Amino Transferase (AST) & $13,62-28,62^{*}$ & $\mathrm{UI} / \mathrm{L}$ & 54,0 & 143,0 & $\mathrm{UI} / \mathrm{L}$ \\
Proteínas totais & $5,49-6,61^{*}$ & $\mathrm{~g} / \mathrm{dl}$ & 5,5 & 4,8 & $\mathrm{~g} / \mathrm{dl}$ \\
Albumina & $2,96-3,62^{*}$ & $\mathrm{~g} / \mathrm{dl}$ & 2,7 & 2,1 & $\mathrm{~g} / \mathrm{dl}$ \\
Globulina & $2,4-3,12^{*}$ & $\mathrm{~g} / \mathrm{dl}$ & 2,8 & 2,7 & $\mathrm{~g} / \mathrm{dl}$ \\
Creatina Quinase (CK) & $41,45-181,77^{*}$ & $\mathrm{UI} / \mathrm{L}$ & 1145,0 & 1352,0 & $\mathrm{UI} / \mathrm{L}$ \\
\hline
\end{tabular}

* Valores de referência para a espécie segundo Oliveira et al. (2018). Fonte: Autores.

Com relação aos resultados obtidos da análise bioquímica sérica comparativa dos dias D0 e D4, observou-se normalização da concentração de ureia, aumento considerável de Alanina Amino Transferase (ALT) e Aspartato Amino 
Transferase (AST), hipoproteinemia por hipoalbuminemia e Creatina Quinase (CK) aumentada em mais de dez vezes o valor de referência.

A antibioticoterapia foi realizada com Amoxicicilina Sódica + Clavulanato de Potássio (Doxaclin® $14 \mathrm{mg} / \mathrm{kg}$, IV, BID por 11 dias. Blau Farmacêutica S.A.. São Paulo, SP, Brasil), Metronidazol (Nidazofarma® 10 mg/kg, IV, BID por 3 dias. Farmace, Barbalha, CE, Brasil) e Sulfato de Amicacina (Sulfato de Amicacina ${ }^{\circledR} 20 \mathrm{mg} / \mathrm{kg}$ IV, SID, por 10 dias. Novafarma, Anápolis, GO, Brasil). Foram administrados ainda Hidrocortisona (Hidrocortisona ${ }^{\circledR} 0,5 \mathrm{mg} / \mathrm{kg}$ IV, por 5 dias. Blau Farmacêutica S.A., São Paulo, SP), Dipirona Sódica (D-500® $25 \mathrm{mg} / \mathrm{kg}$ IM, por 12 dias. Zoetis, Campinas, SP, Brasil) e Metadona (MYTedom ${ }^{\circledR}$ 0,2 mg/kg, IM, por 12 dias. Cristália, Itapira, SP, Brasil).

No quinto dia de internação o paciente foi submetido a novo procedimento anestésico para realização de curativos, colocação de sonda nasogástrica e fixação de acesso intraósseo (Figura 2). Para tal, utilizou-se o mesmo protocolo anestésico já citado anteriormente, assim como as mesmas doses e vias de administração. Devido a grave hipotensão (PAM $40 \mathrm{mmHg}$ ) foi realizada administração intravenosa em bolus de Sulfato de Efedrina (Efedrin ${ }^{\circledR} 0,1 \mathrm{mg} / \mathrm{kg}$. Cristália, Itapira, SP, Brasil) e Atropina Sulfato (Atropina ${ }^{\circledR} 0,25 \mathrm{mg} / \mathrm{kg}$. Hipolabor, Sabará, MG, Brasil) e manutenção em infusão contínua intravenosa de Hemitartarato de Norepinefrina (Hyponor ${ }^{\circledR} 0,1 \mathrm{mcg} / \mathrm{kg} / \mathrm{min}$. Hypofarma, Ribeirão das Neves, MG, Brasil) em solução de Ringer Lactato ${ }^{(10} \mathrm{ml} / \mathrm{kg} / \mathrm{h}$. Halexistar, Goiânia, GO, Brasil).

Figura 2. Tamanduá-bandeira (Myrmercophaga tridactyla) resgatado do Pantanal Mato-grossense após realização do segundo procedimento anestésico para realização de curativos no Hospital Veterinário da Universidade Federal do Mato Grosso campus Cuiabá.

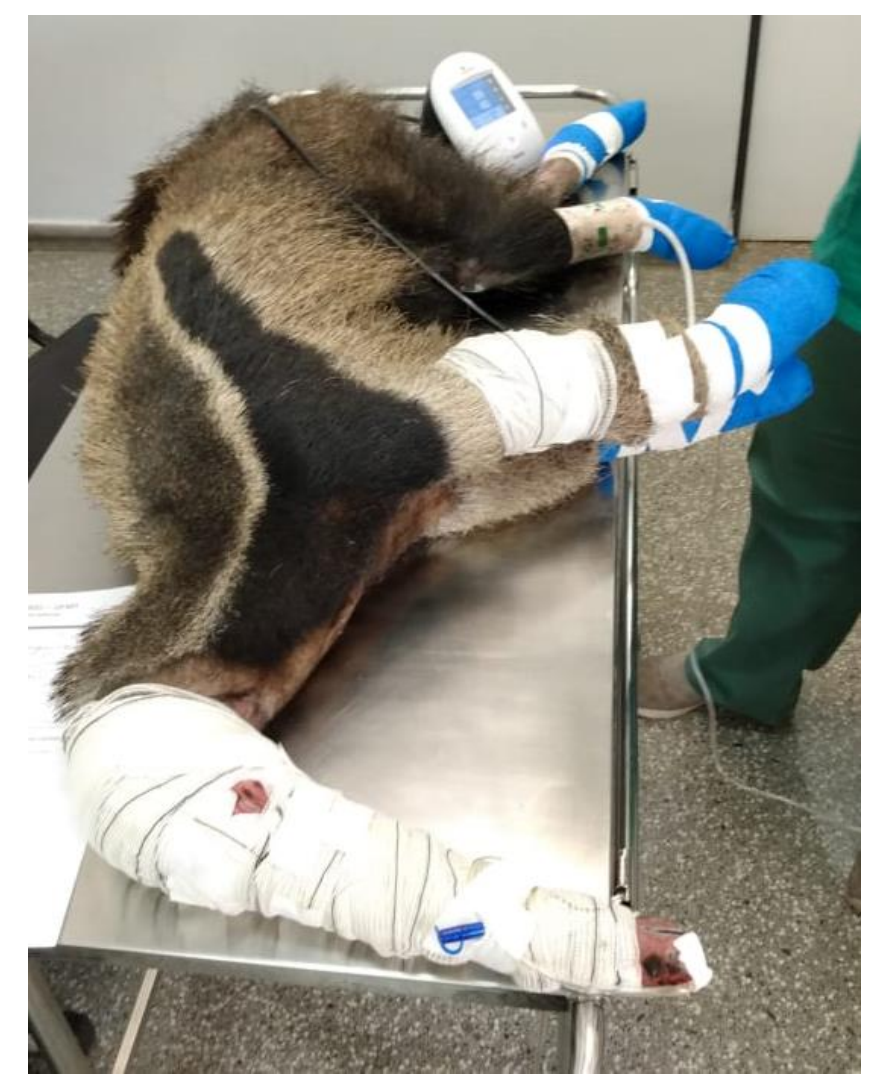

Fonte: Hospital Veterinário da Universidade Federal do Mato Grosso - campus Cuiabá (2020).

Durante os 5 primeiros dias que o paciente permaneceu internado sob cuidados intensivos, realizou-se nutrição microenteral, TID, com $1 \mathrm{ml}$ de Glicopam ${ }^{\circledR}$ (Vetnil, Louveira, SP, Brasil) diluídos em $49 \mathrm{ml}$ de Ringer com Lactato® 
(Halexistar, Goiânia, GO, Brasil). Após a colocação de sonda nasoesofágica, administrou-se concentrado hipercalórico (Nutralife ${ }^{1} 10$ medidas/250ml. Vetnil, Louveira, SP, Brasil) e repositor de eletrólitos (Eletrolítico®, TID, por 7 dias. Vetnil, Louveira, SP, Brasil).

Nos dias seguintes houve melhora do quadro clínico, sendo retirada a infusão contínua de vasopressor. Porém, apesar da resposta positiva ao tratamento empregado, o animal foi encontrado em óbito no décimo segundo dia de internação, não sendo possível realizar o protocolo de ressuscitação cardiopulmonar (RCP).

\section{Resultados e Discussão}

De acordo com dados de um estudo que descreveu as alterações patológicas de 99 tamanduás de cativeiro e vida livre em um período de 24 anos (1994-2018) dos estados brasileiros de São Paulo, Minas Gerais, Bahia, Mato Grosso do Sul e Amazonas os traumas por queimaduras ocupam o segundo lugar de causas mais frequentes de óbitos em tamanduás no Brasil, ficando atrás apenas de lesões por atropelamentos (Arenales et al., 2020).

Queimaduras por calor seco provocam a dessecação e carbonização dos tecidos por proximidade ou contato direto a fontes de calor (Ginn et al., 2007). Este tipo de lesão foi observado no animal relatado neste caso, tendo como causa os incêndios no Pantanal.

De acordo com o nível de dano provocado no tecido, as queimaduras em mamíferos podem ser classificadas em primeiro grau, segundo grau superficial e profundo, terceiro grau e quarto grau. Esta classificação é baseada em características macroscópicas e microscópicas de achados histopatológicos (Wohlsein et al., 2016). Não foi realizada análise histopatológica dos tecidos carbonizados para uma adequada classificação das lesões, porém as características macroscópicas dos ferimentos como eritema difuso, umidade, presença de bolhas e edema permitem inferir que o animal apresentava queimadura de segundo grau profundo na face (Wohlsein et al., 2016).

Merck \& Miller (2013) afirmam que em casos que há queimadura em áreas como rosto, olhos, orelhas, períneo ou pés são considerados mais graves do que aqueles em que outras áreas do corpo. Haja vista que lesões nessas regiões têm o potencial de desfiguração grave, perda de função e dor intensa. Além disso, o envolvimento de aproximadamente $50 \%$ da superfície corporal é considerado incompatível com a vida. No presente relato o animal apresentava queimaduras nos quatro membros e na face, sendo considerado um caso grave e com prognóstico ruim, pois possuía cerca de $63 \%$ de sua superfície corpórea queimada, de acordo com os valores adaptados da "regra de 9" para cálculo de acometimento de superfície corpórea em animais (Wohlsein et al., 2016).

Os achados do eritrograma concordam com o descrito por Barbosa et al. (2009), onde foi relatado diminuição nos valores de hemácias, hemoglobina e hematócrito em humanos acometidos por queimaduras. As possíveis causas atribuídas a esse evento são perda volume sanguíneo no momento dos curativos e debridamento das feridas e aglutinação de eritrócitos nos tecidos devido a redução do fluxo sanguíneo. Os resultados obtidos no eritrograma também concordam com os achados de Magalhães et al, (2022) onde foram observados graus de anemias de moderado a intenso em 6 tamanduás-bandeira com queimaduras térmicas graves.

As lesões provocadas por queimadura de segundo grau profundas são caracterizadas por edema perivascular grave, rico em proteínas, justificando a hipoproteinemia sérica descrita no relato, devido ao extravasamento para o espaço intersticial (Madea \& Schmidt, 2003). Queimaduras de terceiro grau induzem a coagulação em vasos sanguíneos dérmicos, trombose, vasculite subcutânea e hemorragia de vasos sanguíneos profundos com subsequente necrose e edema subcutâneo (Hedlund, 2014), caracterizando a anemia severa notada desde o primeiro hemograma realizado no D0, bem como a necessidade de alta demanda celular para reparo tecidual, comprovada com o desvio à esquerda degenerativo. 
Deve-se considerar que além dos danos causados pelo contato direto com o fogo propriamente dito, os incêndios produzem grande quantidade de fumaça e liberação de gases tóxicos, agentes esses que causam lesões caracterizadas pelo aumento severo da permeabilidade vascular, aderência e demarginação intravascular de neutrófilos (Enkhbaatar et al., 2016). Estudos realizados em ovelhas com indução de queimaduras e inalação de fumaça em ambientes controlados mostraram que as lesões foram caracterizadas por um aumento de neutrófilos no sangue periférico de até cerca de 300\% (Rehberg et al., 2013).

Harvey (2012) afirma que a presença do desvio degenerativo em animais domésticos com distúrbio inflamatório indica que o estímulo para a liberação de neutrófilos pela medula óssea é maior do que o estoque de neutrófilos maduros. A magnitude de um desvio à esquerda em resposta à inflamação pode variar de discreto aumento de bastonetes até graves desvios à esquerda com presença de metamielócitos e mielócitos, justificando não só o aumento de neutrófilos observados no segundo hemograma do paciente, mas também o desvio degenerativo observado no D0.

A presença de grande quantidade de metamielócitos nos leucogramas indica que queimaduras de segundo grau profundas, em mais de 50\% de superfície corpórea são capazes de provocar aumento do consumo e transmigração de células em níveis exacerbados (Harvey, 2012). Comparando o leucograma do D0 com o do D4, apesar de mantido o desvio à esquerda acentuado, nota-se melhor distribuição nos valores de metamielócitos, bastonetes e segmentados, indicando melhora da função de maturação medular.

O aumento de plaquetas está associado a estímulos inflamatórios, liberação de epinefrina e algumas formas de doenças mieloproliferativas (Baker, 2015) portanto, infere-se que o intenso estímulo inflamatório provocado pelas lesões de queimadura causou trombocitose no segundo exame.

A presença de desvio à esquerda degenerativo pode ser usada como um fator prognóstico de sobrevida para cães e gatos com morbidades diversas, uma vez que estes apresentam 1,9 e 1,57 vezes, respectivamente, mais probabilidades de morrerem ou serem eutanasiados do que animais com as mesmas doenças, sem desvio (Burton et al., 2013, 2014).

A atividade de AST, ALT e CK foram decorrentes das lesões musculares provocadas pelas queimaduras, porém não se descarta possibilidade de doença hepática aguda concomitante, devido ao protocolo terapêutico empregado ou lesão preexistente. Outras enzimas como Lactato Desidrogenase, troponinas cardíacas e avaliação de mioglobinúria e mioglobina são indicadas para melhor caracterização do dano à musculatura esquelética (Alisson, 2015).

A antibioticoterapia faz-se necessária na abordagem do paciente queimado pois a vasta quantidade de tecido necrótico presente na ferida proporciona um meio excepcional para o crescimento bacteriano (Fossum, 2014). As lesões por queimaduras causam dor moderada a intensa, a depender do grau da queimadura e da área corporal atingida. A abordagem do tratamento da dor deve ser multimodal. A terapia para controle de dor nesses casos deve ser realizada de forma multimodal utilizando-se a associação de analgésicos opioides e anttinflamatórios (Castro et al. 2013).

\section{Conclusão}

Queimaduras de segundo e terceiro graus em tamanduás-bandeira podem cursar com desvio degenerativo, anemia intensa, hipoproteinemia e alterações marcantes de enzimas que avaliam dano muscular. A depender da extensão da lesão e a evolução clínica do paciente o prognóstico é ruim. O impacto das queimadas no Pantanal mato-grossense no ano de 2020 precisa ser avaliado, sendo necessária a implantação de políticas públicas de combate aos incêndios mais eficazes. Tendo em vista a escassez de informações sobre tamanduás-bandeira do bioma Pantanal outros estudos podem ser realizados a partir deste para melhor caracterização dos achados laboratoriais em Myrmecophaga tridactyla com lesões provocadas por queimaduras. 


\section{Referências}

Alisson, R. W. (2015). Detecção laboratorial das lesões musculares. In: Thrall, M. A., Weiser, G., Allison, R. W. \& Campbell T.W. Hematologia e Bioquímica Clínica Veterinária. (2a ed.), (pp. 412-415).: Guanabara Koogan

Arenales, A., Gardiner, C. H., Miranda, F. R., Dutra, K. S., Oliveira, A. R., \& Mol, J. P. S. Pathology of free-ranging and captive Brazilian anteaters (2020). Journal of Comparative Pathology. 180, 55-68, https://doi.org/10.1016/j.jcpa.2020.08.007

Baker, D.C. (2015). Diagnóstico das Anormalidades de Hemostasia. In: Thrall, M A., Weiser, G., Allison, R.W. \& Campbell, T. W. Hematologia e Bioquímica Clínica Veterinária. (2a ed.), (pp. 159-174).: Guanabara Koogan

Barbosa, A. S. A. A., Calvi, S. A. \& Pereira, P. C. M. (2009). Nutritional, immunological and microbiological profiles of burn patients. Journal of Venomous Animals and Toxins including Tropical Diseases, 15(4), 768-777. https://doi.org/10.1590/S1678-91992009000400014

Burton, A. G., Harris, L. A., Owens, S. D., \& Jandrey, K. E. (2014). Degenerative left shift as a prognostic tool in cats. Journal of Veterinary Internal Medicine, 28(3), 912-917. https://doi.org/10.1111/jvim.12338

Burton, A. G., Harris, L. A., Owens, S. D., \& Jandrey, K. E. (2013). The prognostic utility of degenerative left shifts in dogs. Journal of Veterinary Internal Medicine, 27(6), 1517-1522. https://doi.org/10.1111/jvim.12208

Castro, R. J. A., Leal, P. C., \& Sakata, R. K. (2013). Tratamento da dor em queimados. Revista Brasileira de Anestesiologia,63 (1), 154158. https://doi.org/10.1590/S0034-70942013000100013

Duarte, D. P. F., Silva, V. L., Jaguaribe, A. M., Gilmore, D. P. \& Da Costa, C. P. (2003). Circadian rhythms in blood pressure in free-ranging three-toed sloths (Bradypus variegatus). Brazilian Journal of Medical and Biological Research., 36(2), (pp. 273-278). https://doi.org/10.1590/S0100-879X2003000200016.

Enkhbaatar, P., Pruitt, B. A Jr., Suman, O., Mlcak, R., Wolf, S. E., Sakurai, H. \& Herndon, D. N. (2016). Pathophysiology, research challenges, and clinical management of smoke inhalation injury. Lancet. 388(10052):1437-1446. 10.1016/S0140-6736(16)31458-1

Fossum, T. W. (2014). Queimaduras e outras lesões térmicas. Cirurgia de Pequenos Animais. (4a ed.), (pp. 257-261) Elsevier

Ginn, P. E., Mansell, J. E. K. L. \& Rakich, P. M (2007). Skin and appendages. In: Maxie, M. G., Jubb, Kennedy, and Palmer's. Pathology of Domestic Animals. (5a ed.), (pp. 553-781). Saunders/Elsevier

Harvey, J. W. (2011). Chap 6. Evaluation of Leukocytc Disorders. Veterinary hematology: A Diagnostic Guide and Color Atlas. (pp. 122-176). St. Louis, Missouri, USA: Saunders/Elsevier

Hedlund, C. S. (2014). Cirurgia do sistema tegumentar. In: Fossum, T. W. Cirurgia de Pequenos Animais. (5a ed.), (pp. 134-228) Elsevier

Instituto Nacional de Pesquisas Espaciais (INPE). Programa queimadas: Monitoramento dos Focos Ativos por Bioma. Comparação do total de focos ativos detectados pelo satélite de referência em cada mês, no período de 1998 até 23/01/2021. <http://queimadas.dgi.inpe.br/queimadas/portalstatic/estatisticas_estados/>

Libonati, R., DaCamara, C. C., Peres, L. F., Carvalho, L. A. S. \& Garcia, L. C. (2020). Rescue Brazil's burning Pantanal Wetlands. Nature. 588. $217-219$. https://doi.org/10.1038/d41586-020-03464-1

Madea, B. \& Schmidt, P. (2003). Thermische Energie. In: Madea B, ed. Praxis Rechtsmedizin. Befunderhebung, Rekonstruktion, Begutachtung. 170-186 Berlin, Germany: Springer

Magalhães, T. B. S., Mendonça, A. J., Zorzo, C., Morgado, T. O., Corrêa, S. H. R., Palermo, A. L. P., Nardes, E. R. da S., \& Costa, K. S. (2022). Avaliação do perfil hematológico e bioquímico de Tamanduás-bandeira (Myrmecophaga tridactyla) com queimaduras térmicas graves: Relato de seis casos. Research, Society and Development, 11(2), e45911224480. https://doi.org/10.33448/rsd-v11i2.24480

Merck, M. D. \& Miller, D. M. (2013). Burn, electrical, and fire-related injuries. In: Merck, M. D. ed. Veterinary Forensics: Animal Cruelty Investigations. (2a ed.), (pp. 139-150). Ames, IA: Wiley-Blackwell

Miranda, F. (2014). Cingulata (Tatus) e Pilosa (Preguiça e Tamanduás). In: Cubas, Z. S, Silva, J. R \& Catão-Dias, J. L. Tratado de animais selvagens: medicina veterinária. (2a ed.), (pp. 707-722). Roca

Miranda, F., Bertassoni, A. \& Abba, A. M. (2014). Myrmecophaga tridactyla. The IUCN Red List of Threatened Species $2014:$ e.T14224A47441961. https://dx.doi.org/10.2305/IUCN.UK.2014-1.RLTS.T14224A47441961.en.

Miranda, F. R., Chiarello, A. G., Röhe, F., Braga, F. G., Mourão, G. M., \& Miranda, G. H. B... (2015). Avaliação do Risco de Extinção de Myrmecophaga tridactyla Linnaeus, 1758 no Brasil. Processo de avaliação do risco de extinção da fauna brasileira. ICMBio. http://www.icmbio.gov.br/portal/biodiversidade/fauna-brasileira/lista-de-especies/7049-mamiferos-myrmecophaga-tridactyla-tamandua-bandeira.htm

Nucci, D., Marc, L. B., Jimeno, G., Scapini, J. P., \& Masso, R. J. (2014). Valores Hematológicos y Bioquímica Sanguínea en Osos Hormigueros Gigantes (Myrmecophaga tridactyla) Cautivos en Argentina. Edentata 15. (pp 39-51) http://www.xenarthrans.org

Oliveira, E., Vila, L. G., Trentin, T. C., Jubé, T. O., \& Martins, D. B. (2018). Biochemical parameters of the giant anteater (Myrmecophaga tridactyla Linnaeus, 1758) of the Brazilian Cerrado. Pesquisa Veterinária Brasileira, 38(1), 189-194. https://doi.org/10.1590/1678-5150-pvb-5306

Rehberg, S., Yamamoto, Y., Sousse, L. E., Jonkam, C., Zhu, Y., \& Traber, L. D. (2013). Antithrombin attenuates vascular leakage via inhibiting neutrophil activation in acute lung injury. Critical care medicine, 41(12), e439-e446. https://doi.org/10.1097/CCM.0b013e318298ad3a 
Research, Society and Development, v. 11, n. 3, e42811327113, 2022

(CC BY 4.0) | ISSN 2525-3409 | DOI: http://dx.doi.org/10.33448/rsd-v11i3.27113

Ribeiro, P. R. Q., Santos, A. L. Q., Ribeiro, L. A., Souza, T. A. M., Borges, D. C. S., Souza, R. R. \& Pereira S. G. (2016). Movement anatomy of the gluteal region and thigh of the giant anteater Myrmecophaga tridactyla (Myrmecophagidae: Pilosa). Pesq. Vet. Bras., 36(6), 539-544, https://doi.org/10.1590/S0100736X2016000600013

Weiser, G. (2015). Tecnologia laboratorial em Medicina Veterinária; Coleta e processamento de Amostra e Análise das opções de Serviços Laboratoriais. In: Thrall, M. A., Weiser, G., Allison, R. W \& Campbell, T.W. Hematologia e Bioquímica Clínica Veterinária. (2a ed.), (pp. 2-27). Guanabara Koogan

Wohlsein, P., Peters, M., Schulze, C. \& Baumgärtner, W. (2016). Thermal Injuries in Veterinary Forensic Pathology. Vet Pathol. 53(5):1001-17. $10.1177 / 0300985816643368$ 